Address correspondence to: Booki Min, Department of Immunology/NB30, Lerner Research Institute, Cleveland Clinic Foundation, Cleveland, Ohio 44195, USA. Phone: 216.445.3126; Fax: 216.444.8372; E-mail: minb@ccf.org.

1. Dvorak AM, Nabel G, Pyne K, Cantor H, Dvorak $\mathrm{HF}$, Galli SJ. Ultrastructural identification of the mouse basophil. Blood. 1982;59(6):1279-1285.

2. Min B. Basophils: what they 'can do' versus what they 'actually do'. Nat Immunol. 2008;9(12):1333-1339.

3. Karasuyama H, Mukai K, Tsujimura Y, Obata K. Newly discovered roles for basophils: a neglected minority gains new respect. Nat Rev Immunol. 2009;9(1):9-13.

4. Gessner A, Mohrs K, Mohrs M. Mast cells, basophils, and eosinophils acquire constitutive IL-4 and IL-13 transcripts during lineage differentiation that are sufficient for rapid cytokine production. J Immunol. 2005;174(2):1063-1072.

5. Sokol CL, Barton GM, Farr AG, Medzhitov R. A mechanism for the initiation of allergeninduced T helper type 2 responses. Nat Immunol. 2008;9(3):310-318.
6. Siracusa MC, Perrigoue JG, Comeau MR, Artis D. New paradigms in basophil development, regulation and function. Immunol Cell Biol. 2010; 88(3):275-284

7. Min B, et al. Basophils produce IL-4 and accumulate in tissues after infection with a Th2-inducing parasite. J Exp Med. 2004;200(4):507-517.

8. Kim S, Prout M, Ramshaw H, Lopez AF, LeGros G, Min B. Cutting edge: basophils are transiently recruited into the draining lymph nodes during helminth infection via IL-3, but infection-induced Th2 immunity can develop without basophil lymph node recruitment or IL-3. J Immunol. 2010;184(3):1143-1147.

9. Ohnmacht C, Voehringer D. Basophil effector function and homeostasis during helminth infection. Blood. 2009;113(12):2816-2825.

10. Ohnmacht C, Voehringer D. Basophils protect against reinfection with hookworms independently of mast cells and memory Th2 cells. J Immunol. 2010;184(1):344-350

11. Obata $\mathrm{K}$, et al. Basophils are essential initiators of a novel type of chronic allergic inflammation. Blood. 2007;110(3):913-920

12. Wada T, et al. Selective ablation of basophils in mice reveals their nonredundant role in acquired immunity against ticks. J Clin Invest. 2010;
120(8):2867-2875.

13. Poorafshar M, Helmby H, Troye-Blomberg $M$, Hellman L. MMCP-8, the first lineage-specific differentiation marker for mouse basophils. Elevated numbers of potent IL-4-producing and MMCP-8positive cells in spleen of malaria-infected mice. Eur I Immunol. 2000;30(9):2660-2668.

14. Brown SJ, Askenase PW. Amblyomma americanum: requirement for host $F_{C}$ receptors in antibodymediated acquired immune resistance to ticks. Exp Parasitol. 1985;59(2):248-256.

15. Monteiro GE, Bechara GH. Cutaneous basophilia in the resistance of goats to Amblyomma cajennense nymphs after repeated infestations. Ann NY Acad Sci. 2008;1149:221-225.

16. Brown SJ, Galli SJ, Gleich GJ, Askenase PW. Ablation of immunity to Amblyomma americanum by anti-basophil serum: cooperation between basophils and eosinophils in expression of immunity to ectoparasites (ticks) in guinea pigs. J Immunol. 1982;129(2):790-796.

17. Matsuda H, Fukui K, Kiso Y, Kitamura Y. Inability of genetically mast cell-deficient W/Wv mice to acquire resistance against larval Haemaphysalis longicornis ticks. J Parasitol. 1985;71(4):443-448.

18. Iikura $M$, et al. Transendothelial migration of human basophils. J Immunol. 2004;173(8):5189-5195.

\title{
Seeing red: flushing out instigators of niacin-associated skin toxicity
}

\author{
Richard L. Dunbar 1,2,3,4 and Joel M. Gelfand $2,5,6$
}

\begin{abstract}
${ }^{1}$ Department of Medicine, Division of Experimental Therapeutics, ${ }^{2}$ nstitute for Translational Medicine and Therapeutics, ${ }^{3}$ Institute for Diabetes, Obesity, and Metabolism, ${ }^{4}$ The Cardiovascular Institute, ${ }^{5}$ Department of Dermatology, Department of Epidemiology, and Department of Biostatistics, and ${ }^{6}$ Center for Clinical Epidemiology and Biostatistics, University of Pennsylvania, Philadelphia, Pennsylvania, USA.
\end{abstract}

The use of niacin to improve plasma lipid levels and reduce risk of myocardial infarction is limited by noxious skin effects that result from stimulation of G protein-coupled receptor 109A (GPR109A) in skin immune cells. Niacin causes vasodilation, manifest as rubor (redness) of the head and neck, providing a visible sign associated with other, more bothersome skin complaints. The working theory is that niacin provokes Langerhans cells to produce prostaglandin $\mathrm{D}_{2}\left(\mathrm{PGD}_{2}\right)$, stimulating vascular DP1 receptors to cause vasodilation. In this issue of the JCI, Hanson and colleagues raise a serious challenge to this paradigm in showing that the major player in vasodilation is the keratinocyte, which produces $\mathrm{PGE}_{2}$, stimulating $\mathrm{EP}_{2 / 4}$ receptors, shifting the role of the Langerhans $/ \mathrm{PGD}_{2} / \mathrm{DP}_{1}$ pathway to that of an accomplice. They also show that the antipsoriasis drug monomethyl fumarate, itself a GPR109A agonist, provokes vasodilation through the same cells. These efforts bring us one step closer to solving a key limitation of an important cardioprotective drug and reveal that the skin response to niacin is much more complicated than previously thought.

The therapeutic use of niacin has a long and colorful history (1). Remarkably, it has played a critical role in

Conflict of interest: R.L. Dunbar declares conflicts with Abbott and Merck and Company. J.M. Gelfand declares conflicts with Amgen, Abbott, Centocor, Pfizer, Novartis, Merck and Company, and Celgene.

Citation for this article: J Clin Invest. 2010; 120(8):2651-2655. doi:10.1172/JCI44098. preventing a top cause of death in 2 different centuries by 2 seemingly unrelated mechanisms. It eradicated pellagra, a vitamin deficiency disease caused by a chronic lack of niacin (vitamin $\mathrm{B}_{3}$ ) as a source of $\mathrm{NAD}^{+}$. Before niacin was added to the food supply, pellagra was a major cause of morbidity and mortality in the early 20th century. Presumably through its beneficial effects on lipid levels, niacin also prevents myocardial infarction (MI), a public health menace in the early 21st century (2). Statins have justifiably replaced niacin as the first-line therapy for the treatment of hyperlipidemia; however, even statins only reduce coronary heart disease (CHD) risk by about one-quarter, leaving considerable residual risk in vulnerable populations. Thus, niacin is reemerging as an adjunct to statin therapy to reduce the incidence of CHD events even further. Niacin is also recommended as an adjunct to statin therapy to raise HDL cholesterol and lower triglycerides (2). Although niacin has been used for more than 50 years to lower cholesterol levels, we are only now starting to piece together the molecular mechanism by which it does this, following the discovery that $\mathrm{G}$ protein-coupled receptor 109A (GPR109A) is a receptor for niacin that mediates some of its myriad effects (3-5). GPR109A is highly expressed in adipocytes, where stimulation results in the profoundly antilipolytic effect of niacin and, in turn, the acute reduction in levels of plasma free 


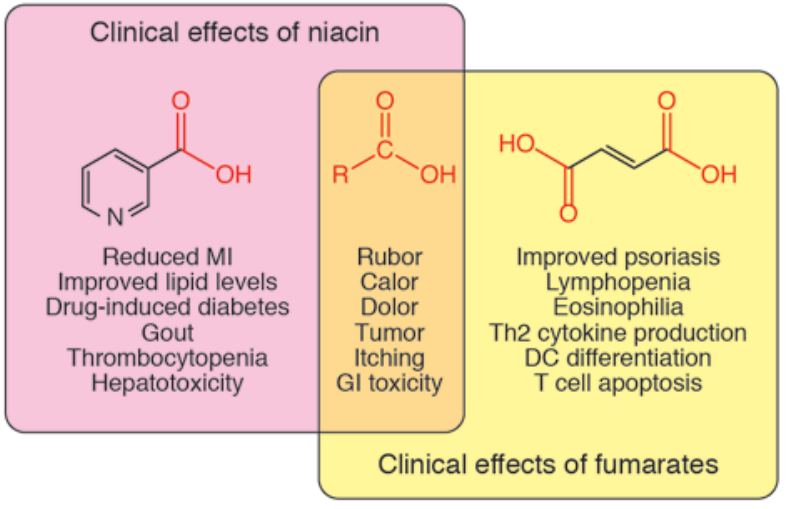

\section{Figure 1}

Overlap of clinical effects of niacin and fumarates. This Venn diagram depicts the known similarities and differences between the clinical effects of niacin (nicotinic acid) and fumarates (fumaric acid). Notably, both are small carboxylic acids (middle). The carboxyl group is an important feature of GPR109A activation and is shared by the known GPR109A agonists. This receptor is responsible for many of the effects of niacin, and its role in psoriasis is now beginning to attract attention. The overlapping symptoms are probably mediated by stimulation of GPR109A, although this has only been shown for rubor. GI, gastrointestinal.

fatty acids (4). Although this effect on free fatty acids has been conveniently assumed to mediate the lipid-altering effects of niacin, the GPR109A agonist acipimox has much weaker effects on lipid levels (6), and a novel GPR109A agonist had no meaningful effects (7). Thus, the mechanisms by which niacin improves plasma lipid levels remain elusive.

\section{Skin toxicity limits niacin use for treatment of dyslipidemia and prevention of MI}

Unfortunately, when used as a pharmaceutical, niacin has more than its share of drug toxicities, including hepatotoxicity, gastric toxicity, glucotoxicity, and, most commonly, skin toxicity. Thus, despite its ability to prevent MI, in practice, niacin is often shunned for its aggressive irritant effects in the skin. Approximately $20 \%$ of niacin-treated subjects drop out of clinical trials (6), and in the clinic, nonadherence and discontinuation are important obstacles to niacin therapy. Skin plays a large role in thermoregulation, but is also the largest immune organ. Pharmacologic doses of niacin disrupt both functions with a vengeance, as part of skin toxicity.

Both deficiency of and exposure to niacin provoke the classical features of dermal toxicity, namely rubor (redness from vasodilation), calor (warmth), dolor (pain), and tumor (swelling). In niacin deficiency, this is referred to as dermatitis. In niacin exposure, these features are transient and are more akin to an acute hypersensitivity syndrome (8) than to dermatitis. In histology sections, niacin exposure is observed to elicit a mononuclear infiltrate within minutes, followed by a neutrophil infiltrate within a few hours that persists for up to 48 hours (9). Clinically, niacin exposure also involves paresthesia (a sensation of tingling, pricking, or numbness) and, perhaps the most irritating symptom, itching. For want of a better word, the full syndrome has gone by the term flushing, referring to the most visible feature, rubor. Although colorful, we are not sanguine about the term flushing, as it fails to capture the full extent of this drug-induced skin toxicity. Indeed, it is not clear whether rubor mediates the other symptoms. Calor may be mediated by rubor, as warmer core blood is brought to the surface, but could also result from increased local metabolism (9). Even so, linkage between rubor and the other irritative symptoms is speculative. We submit that referring to the specific sign flushing as a metonym for symptoms such as skin pain and/or itching invites misunderstanding at best, and diverts attention away from the real clinical problem at worst. We propose the use of flushing should be limited to rubor and think this area would be better served if the broader syndrome of rubor, calor, dolor, tumor, and itching were referred to as niacin-associated skin toxicity to better reflect broad pathologic effects on the skin. Despite the gross and histological complexity of skin toxicity, most animal and clinical studies have focused on rubor and, to a lesser extent, calor. Importantly, experimental models may miss the most irritating symptoms, so we cannot presume that a reduction in rubor will actually translate to a meaningful improvement in tolerability.

\section{Molecular basis of a niacin- associated skin toxicity reaction}

Given the high rates of discontinuation, it is imperative to develop a clinical strategy to suppress niacin-associated skin toxicity without compromising the benefits of the drug if it is to be used to prevent MI on a broader scale. As with the effects on lipid levels, the mechanism underlying niacin-associated skin toxicity has been elusive until fairly recently. Fortuitously, the identification of GPR109A as a receptor for niacin (3-5) has revolutionized the study of the noxious skin effects of the drug. GPR109A is highly expressed in a variety of cells, notably neutrophils, adipocytes, Langerhans cells, keratinocytes, and monocytes (10), and growing evidence suggests that agonizing GPR109A on skin immune cells incites a cascade of events that drives at least rubor (11), if not the other irritative features. Upon stimulation of immune cells, GPR109A promotes activation of phospholipase $A_{2}$, production of arachidonic acid, and, via cyclooxygenase (COX) enzymes, generation of prostaglandin $\mathrm{D}_{2}\left(\mathrm{PGD}_{2}\right)$ and $\mathrm{PGE}_{2}$. These activate $\mathrm{DP}_{1}$ and $\mathrm{EP}_{2 / 4}$ receptors, respectively, which cause relaxation of vascular smooth muscle and, in turn, vasodilation and rubor (11). A fuller lineup of suspected instigators of niacin-associated skin toxicity includes Langerhans cells $(10,12)$, macrophages (13), mast cells (14), and platelets (14).

The working theory points to the Langerhans cell $/ \mathrm{PGD}_{2} / \mathrm{DP}_{1}$ pathway as the prime suspect for rubor, and this pathway has been targeted for discovery and drug therapy $(10,12)$. $\mathrm{A} \mathrm{DP}_{1}$ receptor antagonist, laropiprant, has been developed to mitigate niacin-induced flushing, and is currently marketed in Europe. In clinical trials, laropiprant substantially reduced, but fell far short of eliminating, objectively measured rubor and calor and subject-reported skin symptoms (15). This suggests that, in humans, other pathways beyond the $\mathrm{PGD}_{2} /$ $\mathrm{DP}_{1}$ pathway are important. These results are consistent with a mouse model of niacin-induced rubor in which mice lacking the $\mathrm{DP}_{1}$ receptor had only a partial reduction in rubor (16). These studies led to 


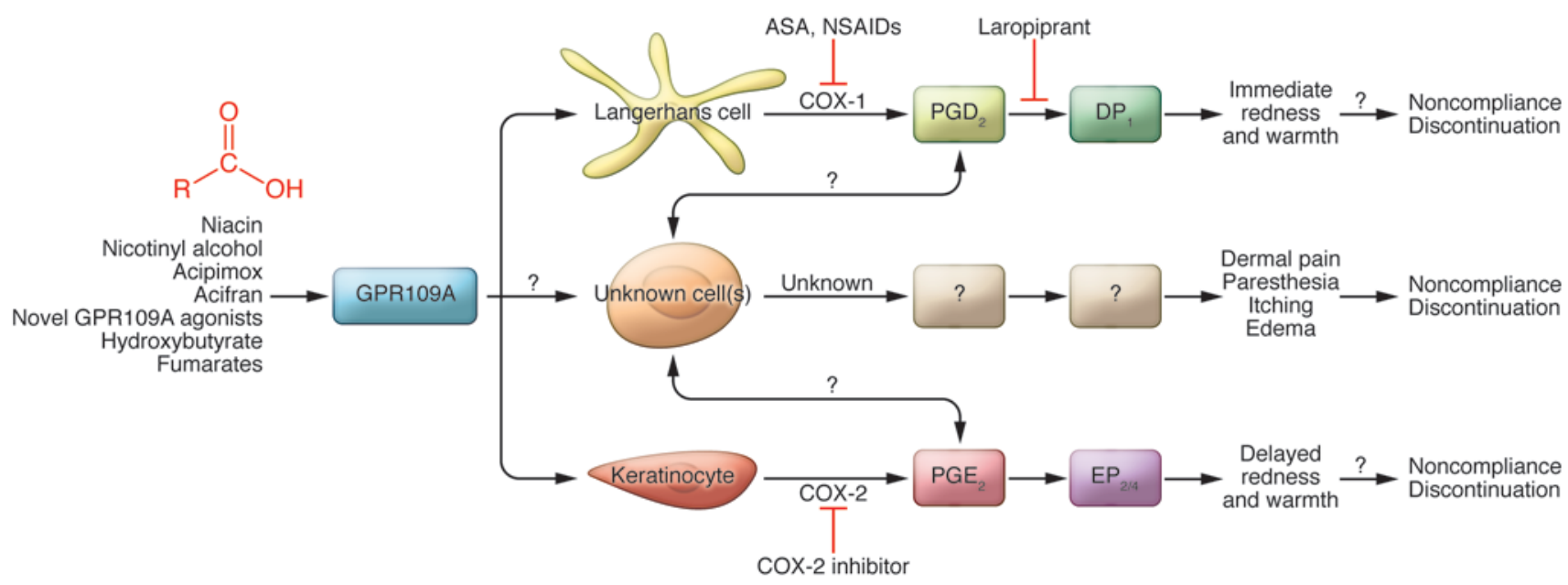

Figure 2

Proposed model of niacin-associated skin toxicity. This diagram illustrates the many missing pieces that contribute to niacin- and GPR109Ainduced skin toxicity. Langerhans cells and keratinocytes cause vasodilation and rubor (redness), and probably calor (warmth), by producing specific prostaglandins under the influence of different COX enzymes. It is not clear, however, that rubor and calor are the symptoms that truly motivate patients to abandon therapy. Other cells may be involved in dolor, tumor, and itching, either in response to GPR109A stimulation or perhaps indirectly via GPR109A-mediated activation of Langerhans cells or keratinocytes. We propose that the latter irritative symptoms, rather than rubor and calor, drive noncompliance with niacin therapy and its discontinuation and suggest that animal and human studies focusing on these phenomena could help to improve drug tolerability.

the hypothesis that niacin-associated skin toxicity, including rubor, is multifactorial and that factors beyond $\mathrm{PGD}_{2}$ are involved in vasodilation and the other skin effects of the drug. Although much progress has been made, the details of how niacin causes rubor and niacin-associated skin toxicity remain a mystery.

\section{Surprisingly, the keratinocyte is the archvillain in niacin-induced rubor among rodents}

In this issue of the JCI, Hanson and colleagues have uncovered a pivotal clue that implicates keratinocytes as the key instigators of the rubefacient properties of GPR109A agonists, and sorted out both the role and the mechanism of Langerhans cells and keratinocytes in rubor (17). First, they showed that mouse and human keratinocytes expressed endogenous GPR109A, resolving prior uncertainty $(10,18)$. They also showed that keratinocyte GPR109A was functional using Gpr109aknockout mice (17).

Next, the authors determined how Langerhans cells and keratinocytes contribute to the time course of rubor. Although rubor can be assessed directly by colorimetry, they used laser Doppler flowmetry to estimate the flux of red blood cells in the skin. This provides a continuous measure of hyperemia, which is an excellent proxy for rubor. As with humans, Hanson et al. detected multiple peaks of hyperemia in wild-type mice: an abrupt but fleeting minor peak followed by a slow-onset, prolonged major peak (17). However, when the mice were manipulated such that their Langerhans cells were eradicated and replaced with GPR109A-deficient Langerhans cells, then no minor peak was observed, although the major peak was retained. This implied that the minor peak depended on intact Langerhans cells, whereas the major peak was caused by GPR109A stimulation on other cells.

To elucidate the cells responsible for inducing the major peak of hyperemia, Hanson and colleagues generated mice that only expressed GPR109A in keratinocytes (17). Whereas the complete Gpr109a knockout lacked both peaks of hyperemia, mice expressing GPR109A exclusively in keratinocytes exhibited the major, delayed peak of hyperemia upon exposure to niacin. Thus, the minor hyperemia peak is a result of GPR109A stimulation on Langerhans cells, whereas the major hyperemia peak originates from GPR109A stimulation on keratinocytes.

Hanson et al. went on to develop robust evidence supporting a new theory of hyperemia. Specifically, their data indicated that upon agonizing GPR109A, a flash of hyperemia occurred almost immediately, as Langerhans cells synthesized $\mathrm{PGD}_{2}$ under the influence of COX-1. This short-lived effect was followed by a delayed but more sustained course of hyperemia, as keratino- cytes synthesized $\mathrm{PGE}_{2}$ under the influence of COX-2. Each peak was suppressible by its respective COX inhibitor (17).

\section{What does this mean for the patient who wants to prevent MI but doesn't tolerate niacin?}

We can speculate as to how the results of Hanson et al. (17) might translate to prevention of niacin-associated skin toxicity in humans. If rubor is upstream of the irritative symptoms, then the solution to niacin intolerance is simply a matter of suppressing the COX enzymes in the right proportion. The authors' work suggests that suppressing the keratinocyte/ COX-2/PGE $2 / \mathrm{EP}_{2 / 4}$ pathway might provide additive relief to suppression of the Langerhans/COX-1/PGD $2 / \mathrm{DP}_{1}$ pathway (17). On the other hand, if rubor and the irritative symptoms are both downstream of GPR109A, but not linked, then suppressing rubor may remain disappointing clinically (15). Unfortunately, there is clinical evidence to suggest that rubor does not track with other irritative skin symptoms. Lai and colleagues attempted to correlate hyperemia (i.e., rubor) by laser Doppler, with subject-reported symptoms of flushing and warmth after treatment with niacin (15). On the first dose, hyperemia correlated tightly with symptoms. Surprisingly, the relationship deteriorated after 3 days of niacin therapy, so that hyperemia flourished 
unabated, but symptoms were at roughly half their initial level. To our knowledge, this is the first compelling evidence that an objectively measured rubor is dissociated and discordant from subjectively reported perception of flushing and warmth in humans. This suggests that symptoms that bother patients are not largely driven by rubor. The matter is complicated by the fact that symptom scales may meld calor (thermoception) with dolor (nociception) into "warmth," making it hard to interpret clinical changes. The disconnect between rubor and irritative symptoms could have important ramifications for drug development, because preclinical work is largely based on hyperemia. If this sign is divorced from the symptoms that most irritate patients, one could unwittingly develop a drug that reduces rubor without mitigating the more bothersome symptoms of niacinassociated skin toxicity. Consider dermal pain and itching. Is vasodilation enough to explain these? Does vigorous activation of keratinocytes, Langerhans cells, both, or neither drive these? Does some other resident or infiltrating cell mediate the irritative symptoms by a completely different process? Without knowing how the most troubling symptoms fit in to the mystery, it is hard to predict the clinical impact of blocking rubor.

\section{The antipsoriatic fumarates act as niacin analogs}

Hanson and colleagues also raise several interesting questions by showing that an antipsoriasis drug drives a hyperemia response indistinguishable from that driven by niacin in mice (17). As with niacin, fumaric acid esters (FAEs; also known as fumarates) have a long history in medicine. Fumarates have been used for the treatment of psoriasis since 1959. Currently, they are approved to treat severe psoriasis in Germany, but are not approved in the United States. Fumarates have potent immune-modifying properties (Figure 1 and ref. 19) and are under investigation for use in the treatment of other immunemediated diseases, such as multiple sclerosis. Like niacin, fumarates are small carboxylic acids. Both require slow dose escalation to minimize dermal and gastric toxicity (Figure 1), and about $30 \%$ of patients stop therapy as a result of these side effects. It is now clear that fumarates stimulate the GPR109A receptor (18), so our emerging understanding of niacin-associated skin toxicity can and should be extended to fumarate therapy. The group that reported that fumarate agonizes GPR109A showed that skin from psoriasis patients expressed GPR109A more abundantly than did controls, and speculated that GPR109A is implicated in the disease and perhaps its remediation by fumarate (18).

Interestingly, although both niacin and fumarates agonize GPR109A, they have divergent clinical benefits and toxicities (Figure 1). For example, niacin is not known to improve psoriasis, and fumarates are not known to improve lipid levels (19). In clinical trials, fumarates often cause lymphopenia and eosinophilia, side effects not seen with niacin. Therefore, GPR109A-associated skin toxicity could be an off-target effect for both drugs in their respective diseases. If so, the extensive bag of tricks developed to enhance niacin tolerability (such as skillfully selected COX inhibitors and laropiprant) may apply to fumarate treatment. Another worthy question is whether fumarates, acting as GPR109A agonists, have beneficial effects on lipid levels or cardiovascular risk. Given that psoriasis itself is associated with higher risk for cardiovascular disease and dyslipidemia $(20,21)$, this is not just an academic question; if confirmed, it might commend the broader use of fumarates as antipsoriatic drugs.

\section{Next steps: thinking beyond rubor}

The work by Hanson and colleagues represents a major step forward in understanding rubor from GPR109A agonism (17) and challenges the longstanding paradigm that $\mathrm{PGD}_{2}$ is the major villain in rubor. Although both $\mathrm{PGD}_{2}$ and $\mathrm{PGE}_{2}$ are now convincingly linked to rubor in mice, the new findings need to be translated into humans, since it is not entirely clear that rubor or even calor really motivate certain patients to swear off niacin. Although it is conceivable that $\mathrm{PGD}_{2}$ and/or $\mathrm{PGE}_{2}$ cause skin pain and itching, our instinct is that there is still more to the scene than meets the eye. Activation of the immune system is often an all-hands-on-deck proposition, and the concept that only a few resident cell types acting in isolation mediate such a diverse range of noxious effects strikes us as unlikely. Given that GPR109A is expressed by a variety of immune cells that are present in the skin and its inflammatory infiltrate, we find it likely that other cells and the mediators they produce will provide a more detailed explanation for the full expression of the niacin-associated skin toxicity reaction. Our proposed model of GPR109A-associated skin toxicity is presented in Figure 2. Most clinical work has concentrated on the COX-1/PGD $/$ $\mathrm{DP}_{1}$ pathway. It is well known that COX-1 inhibition with aspirin and NSAIDs partially inhibits skin toxicity. Downstream, laropiprant was developed to disrupt the interaction of $\mathrm{PGD}_{2}$ with $\mathrm{DP}_{1}$ receptors, which reduces vasodilation and thereby rubor. However, if COX-2 activation is the major villain in rubor, one would predict that enhancing COX-2 inhibition would improve tolerability further. Although one could easily test this in a proof-of-concept study in humans, using this approach during chronic therapy would be problematic, as COX-2 inhibitors may increase CHD events (22). However, based on the results of Hanson et al. (17), inhibition of $\mathrm{PGE}_{2}$ synthesis or blockade of the EP receptors might provide a novel approach to even more effective prevention of niacin-associated skin toxicity.

We find value in research into the molecular mechanisms of niacin-associated skin toxicity and in the development of drugs, such as laropiprant, to mitigate them. Even if laropiprant does not fully eliminate skin toxicity, merely lowering the intensity of symptoms could help extend niacin to a broader section of the population at risk for MI. We eagerly await the results of a large clinical trial on the efficacy of niacin plus laropiprant added to a statin on hard cardiovascular outcomes (http://www.clinicaltrials.gov/ct2/ show/NCT00461630). We commend Hanson and colleagues for their major contribution to helping solve the mystery of GPR109A-associated skin toxicity (17) and hope to see their insights based on murine studies translated into humans.

\section{Acknowledgments}

Funding for this work was provided by NIH grants K23HL091130 (R.L. Dunbar), SCCOR P50-HL-083799 (R.L. Dunbar), and RO1HL089777 (J.M. Gelfand) from the National Heart, Lung, and Blood Institute; by the Psoriasis Research Foundation in Honor of Herman Beerman (J.M. Gelfand); and by NIH Clinical and Translational Science Award UL1-RR-024134 from the National Center for Research Resources.

Address correspondence to: Richard L. Dunbar, Penn Presbyterian Medical Center, Andrew Mutch Building Rm. 107, Experimental Therapeutics Division, Institute for Translational Medicine and Therapeutics, 51 N. $39^{\text {th }}$ Street, Philadelphia, Pennsylva- 
nia 19104, USA. Phone: 215.662.9024; Fax: 215.243.1519; E-mail: richard.dunbar@ uphs.upenn.edu.

1. Carlson LA. Nicotinic acid: the broad-spectrum lipid drug. A 50th anniversary review. J Intern Med. 2005;258(2):94-114.

2. National Cholesterol Education Program (NCEP) Expert Panel on Detection, Evaluation, and Treatment of High Blood Cholesterol in Adults (Adult Treatment Panel III). Third Report of the National Cholesterol Education Program (NCEP) Expert Panel on Detection, Evaluation, and Treatment of High Blood Cholesterol in Adults (Adult Treatment Panel III) final report. Circulation. 2002;106(25):3143-3421.

3. Wise A, et al. Molecular identification of high and low affinity receptors for nicotinic acid. J Biol Chem. 2003;278(11):9869-9874.

4. Tunaru S, et al. PUMA-G and HM74 are receptors for nicotinic acid and mediate its anti-lipolytic effect. Nat Med. 2003;9(3):352-355.

5. Soga T, et al. Molecular identification of nicotinic acid receptor. Biochem Biophys Res Commun. 2003;303(1):364-369.

6. Birjmohun RS, Hutten BA, Kastelein JJP, Stroes ESG. Efficacy and safety of high-density lipoprotein cholesterol-increasing compounds: a metaanalysis of randomized controlled trials. J Am Coll Cardiol. 2005;45(2):185-197.

7. Lai E, et al. Effects of a niacin receptor partial ago- nist, MK-0354, on plasma free fatty acids, lipids, and cutaneous flushing in humans. J Clin Lipidol. 2008;2(5):375-383

8. Basketter DA, Wilhelm KP. Studies on non-immune immediate contact reactions in an unselected population. Contact Derm. 1996;35(4):237-240

9. English JS, Winkelmann RK, Louback JB, Greaves MW, MacDonald DM. The cellular inflammatory response in nicotinate skin reactions. BrJ Dermatol. 1987;116(3):341-349.

10. Maciejewski-Lenoir D, Richman JG, Hakak Y, Gaidarov I, Behan DP, Connolly DT. Langerhans cells release prostaglandin D2 in response to nicotinic acid. J Invest Dermatol. 2006;126(12):2637-2646

11. Gille A, Bodor ET, Ahmed K, Offermanns S. Nicotinic acid: pharmacological effects and mechanisms of action. Annu Rev Pharmacol Toxicol. 2008;48:79-106.

12. Benyó Z, Gille A, Bennett CL, Clausen BE, Offermanns S. Nicotinic acid-induced flushing is mediated by activation of epidermal Langerhans cells. Mol Pharmacol. 2006;70(6):1844-1849.

13. Meyers CD, Liu P, Kamanna VS, Kashyap ML. Nicotinic acid induces secretion of prostaglandin D2 in human macrophages: an in vitro model of the niacin flush. Atherosclerosis. 2007;192(2):253-258.

14. Papaliodis D, et al. Niacin-induced "flush" involves release of prostaglandin D2 from mast cells and serotonin from platelets: evidence from human cells in vitro and an animal model. J Pharmacol Exp Ther. 2008;327(3):665-672.

15. Lai E, et al. Suppression of niacin-induced vasodila- tion with an antagonist to prostaglandin D2 receptor subtype 1. Clin Pharmacol Ther. 2007;81(6):849-857.

16. Benyo Z, et al. GPR109A (PUMA-G/HM74A) mediates nicotinic acid-induced flushing. J Clin Invest. 2005;115(12):3634-3640

17. Hanson J, et al. Nicotinic acid- and monomethyl fumarate-induced flushing involves GPR109A expressed by keratinocytes and COX-2-dependent prostanoid formation in mice. J Clin Invest. 2010;120(8):2910-2919.

18. Tang H, Lu JY-L, Zheng X, Yang Y, Reagan JD. The psoriasis drug monomethylfumarate is a potent nicotinic acid receptor agonist. Biochem Biophys Res Commun. 2008;375(4):562-565.

19. Wain EM, Darling MI, Pleass RD, Barker JN, Smith $\mathrm{CH}$. Treatment of severe, recalcitrant, chronic plaque psoriasis with fumaric acid esters: a prospective study. Br J Dermatol. 2010;162(2):427-434

20. Gelfand JM, Neimann AL, Shin DB, Wang X, Margolis DJ, Troxel AB. Risk of myocardial infarction in patients with psoriasis. JAMA. 2006; 296(14):1735-1741

21. Mehta NN, Azfar RS, Shin DB, Neimann AL, Troxel AB, Gelfand JM. Patients with severe psoriasis are at increased risk of cardiovascular mortality: cohort study using the General Practice Research Database. Eur Heart J. 2010;31(8):1000-1006.

22. Grosser T, Fries S, FitzGerald GA. Biological basis for the cardiovascular consequences of COX-2 inhibition: therapeutic challenges and opportunities. J Clin Invest. 2006;116(1):4-15

\title{
PIK3CA and KRAS mutations predict for response to everolimus therapy: now that's RAD001
}

\author{
Morassa Mohseni and Ben Ho Park
}

Breast Cancer Research Program, The Sidney Kimmel Comprehensive Cancer Center at Johns Hopkins, Baltimore, Maryland, USA.

\begin{abstract}
Targeted cancer therapeutics can be effective when patients are preselected to maximize the chance of response. Increasingly, molecular markers such as oncogenic DNA mutations are being exploited to help guide patient preselection. These DNA lesions can predict for either a positive or negative response to a given drug. Finding such predictive biomarkers is an ongoing challenge. New work by Di Nicolantonio and colleagues in this issue of the JCI demonstrates that PI3K catalytic $\alpha$ subunit (PIK3CA) mutations can sensitize cancer cells to the mammalian target of rapamycin (mTOR) inhibitor everolimus. In addition, they show that the concurrent presence of PIK3CA mutations and mutations in either KRAS or BRAF predict for resistance to this drug. These data suggest that mTOR inhibitors currently in use will be ineffective against cancers that have a mutation in either KRAS or BRAF despite having PI3K/AKT/mTOR pathway activation.
\end{abstract}

In the past few decades, developers of new anticancer therapies have moved away from cytotoxic drugs that simply target the proliferative hallmark of all cancer cells. Cur-

Conflict of interest: Ben Ho Park is a consultant for, and has received research funding from, GlaxoSmithKline and is a consultant for Horizon Discovery Ltd.

Citation for this article: J Clin Invest. 2010; 120(8):2655-2658. doi:10.1172/JCI44026. rently, targeted therapies dominate cancer drug development with the aim of blocking the growth and spread of cancer by interfering with specific molecules involved in the progression of a given tumor. One of the most successful targeted anticancer therapies developed is the kinase inhibitor imatinib, which targets the product of the $B C R-A B L$ oncogene that drives disease in all patients with chronic myeloid leuke- mia (CML) (1). However, for most targeted therapies, only a subset of the patients predicted to respond do so. For example, EGFR-directed therapies were thought to inhibit the growth of non-small-cell lung cancers with EGFR overexpression, but only those cancers with certain activating EGFR mutations respond to these small molecule inhibitors $(2,3)$. It has therefore become critically important to develop predictive biomarkers for patients who are likely to respond to a given therapy and, equally important, for those who will not. As an example, testing for KRAS mutations has become mandatory for colorectal cancer patients receiving EGFR-directed therapies because the presence of KRAS mutations predicts for resistance to this class of drugs (4). In this issue of the JCI, Di Nicolantonio and colleagues have now uncovered mutations that seem to predict for response to the anticancer drug everolimus, which targets mammalian target of rapamycin (mTOR) (5). 